\title{
ON THE EVOLUTIONARY STAGE OF V448 CYG
}

(Abstract)

\author{
M. KUMSIASHVILY \\ Astronomical Council of the Academy of Science, U.S.S.R.
}

The light-curve of the massive binary star V448 Cyg has been investigated. The mass of the components and rate of mass exchange is estimated; the evolutionary stage of the star is determined. It appears that in V448 Cyg the mass exchange possibly occurs for the second time. 